\title{
Reliable Facility Systems Based on the p-median Problem Subject to Edge Failures
}

\author{
Zongtian Wei ${ }^{1, a}$ Shenggui Zhang ${ }^{2, b}$ and Nannan $Q i^{3, c}$ \\ ${ }^{1}$ Department of Applied Mathematics, Northwestern Polytechnical University,
}

Xi'an, 710072, China

${ }^{2}$ The State Key Laboratory for Manufacturing Systems Engineering (Xi'an Jiao Tong University), Xi'an, 710049, China

${ }^{3}$ Science and Technology on EO-control Laboratory, Luoyang Institute of Electro-optic Equipment, Luoyang, 471009, China

a ztwei@xauat.edu.cn, b sgzhang@nwpu.edu.cn, xuguang01@yahoo.com.cn

Keywords: facility system, reliability, edge failure, location problem

\begin{abstract}
The reliability of a facility system is to a large degree adversely affected by the edge facility failures. In this paper, we consider the design of reliable facility systems based on the classical p-median problem subject to edge failures. A reliable model is formulated and a scenario based algorithm is applied for computing an instance. The results show that the reliability of a facility system will increase when considering the edge facility failures in locating decision.
\end{abstract}

\section{Introduction}

Church and Scaparra [1] defined critical infrastructure as those elements which are necessary for life line support and safety. They include such systems as communication systems, transportation systems, water and sewer systems, health services facilities, etc. Each of these systems has unique properties that may define specific issues in operation and management in order to provide a consistent and continuing level of operation.

Every facility system in operation maybe faces various disruptions and facility system disruptions can have significant physical costs. Therefore, Reliability is receiving high attention in the design of facility systems [2].

The reliability of a facility system is the probability that all suppliers are operable [3]. This concept is introduced from the network reliability theory. Generally speaking, the key difference between them is that the network reliability is primarily concerned with connectivity; it considers the cost of constructing the network but not the cost that results from a disruption, whereas the facility system reliability considers both types of costs and generally assume connectivity after a disruption [4].

In location science, the problem of locating p-supply facilities that yields the smallest weighted distance is called the p-median problem (PMP). The PMP has been the subject of considerable research, starting with the theorems of Hakimi [5], the first heuristic of Teitz and Bart [6] and an integer linear programming model of Revelle and Swain [7]. Church [8] provided a detailed summary of different approaches for solving the PMP.

There is a mature literature on reliable network design and analysis under component failures. Unfortunately, so far we have not found the explicit study of reliable facility systems design subject to edge failures. In fact, the reliability, and hence the efficiency, of a facility systems is to a large degree adversely affected by failures of the edges. Thus the network-based facility system reliability models to be investigated are more practical and closer to the reality of facility system management.

In this paper, we will mainly consider the design of reliable facility systems based on the classical p-median problem when subject to edge disruptions. The remainder of the paper is organized as follows. In Section 2, we formulate the reliability model based on the p-median problem and edge 
failures. We use a scenario based algorithm to compute an instance and give the reliable facility location solutions in Section 3. Section 4 is a summary of this paper.

\section{The Reliable Facility Location Models}

The p-median Problem. The PMP locates $p$ facilities to minimize the demand-weighted total (or average) distance between demands and the nearest facility. The following are the notations for our formulations.

Sets

$\boldsymbol{I}$ : set of customers, indexed by $i$.

$\boldsymbol{J}$ : set of potential facility locations, indexed by $j$.

Parameters

$\boldsymbol{h}_{\boldsymbol{i}}$ : demand at customer $i \in I$.

Decision variables

$$
\begin{gathered}
X_{j}= \begin{cases}1, & \text { if a facility is established at location } j \in J \\
0, & \text { otherwise }\end{cases} \\
Y_{i j}= \begin{cases}1, & \text { if a facility } j \in J \text { serves } \text { c ustomer } i \in I \\
0, & \text { otherwise }\end{cases}
\end{gathered}
$$

The PMP model is

$$
\begin{array}{lll}
\text { Min } & \sum_{j \in J} \sum_{i \in I} h_{i} d_{i j} Y_{i j} & \\
\text { s.t. } & \sum_{j \in J} Y_{i j}=1 & \forall i \in I \\
& Y_{i j}-X_{j} \leq 0 & \forall i \in I, \quad j \in J \\
& \sum_{j \in J} X_{j}=P & \\
& X_{j} \in\{0,1\} & \forall j \in J \\
& Y_{i j} \in\{0,1\} & \forall i \in I \quad \forall j \in J
\end{array}
$$

The objective function (1) minimizes the total demand-weighted distance between customers and their assigned facilities. Constraints (2) require that each customer to be served by exactly one facility. Constraints (3) require that any customer can't be assigned to an unopened facility. Constraint (4) requires $p$ facilities to be opened and Constraints (5) and (6) require the location variables and the assignment variables to be binary.

The Definition of Cost Reliability. Snyder et al. [9, 10] studied the reliability of some uncapacitated, fixed-charge and deterministic demand location problems subject to disruptions. They considered the "vertex" facility failures only and measured the facility system's reliability by the increase of cost after disruptions. They pointed out that in most cases the reliability increase obviously with a small amount of extra cost. Unfortunately, they didn't define the reliability explicitly.

Since the most facility systems are established by the optimal solutions of location problems and the main objective is minimizing the operation cost, we then define the cost reliability of such systems as follows.

Definition: Let $(D, f)$ be the mathematical model of a location problem $P$, where $D$ and $f$ represent the constraints and the cost objective function, respectively. Assume $S$ is a facility system based on the optimal solution of $P$, if $D$ is changed to be $D$ 'because of some of the system's parameters have changed or some facilities have failed, the ratio of the optimal objective function value of $(D, f)$ and $\left(D^{\prime}, f\right)$ is called the cost reliability, or the relative reliability of the system under the constraints $D^{\prime}$.

In this paper, the discussion of facility systems reliability will be in the framework of this definition. 
The Reliable PMP Models. We view a facility system as a weighted connected simple graph $G=(V, E, H, D)$, where $V=I \cup J$;

$E$ is the edge set with the edges denoting goods or information paths; $H$ is the vertex weight set with the weight $h_{i}$ of vertex $i$, denoting the demand of customer $i$, and $D$ is the edge weight set with the weight $d_{i j}$ of edge $(i, j)$, denoting the length (e.g., distance) between $i$ and $j$ under the existing conditions. By $d_{i j}$ we also denote the shortest path length (or distance) between $i$ and $j$ if $(i, j) \notin E$. Evidently, $0 \leq d_{i j}<+\infty$.

Let $F \subseteq E$ be the target edge (the potential loss edge) set of an attack. Assume that an attacker can hit each edge in $F$ at most once and that the edges in $F$ will be hit simultaneously. Furthermore, we assume that the edge failures are independent, that is, a failed edge does not affect any other edge.

Obviously, any $C(\subseteq J)$ such that $|C|=p$ represents a feasible solution of the PMP and there are $C_{|J|}^{p}$ location formulae totally. Let $S$ be the set of all feasible solutions. Then any $s \in S$ has $p$ vertices and represents a subset of $J$. The reliable $p$-median problem (RPMP) model then selects a solution which minimizes the total demand-weighted distance between customers and their assigned facilities from $S$ subject edge failures.

For any $s \in S$, denote the intersection of the edges in the delivery routes (without edge failures, for example, see the red color edges in the figure 1) and $F$ by $E_{s}$, then any $F_{s} \subseteq E_{s}$ represents an edge failure scenario. Assume the failure probability of $e \in F$ is $p_{e}$, then the occurrence probability of $F_{s}$ is $p_{F_{s}}=\prod_{e \in F_{s}} p_{e} \prod_{e \in E_{s} \backslash F_{s}}\left(1-p_{e}\right)$.

The Decision variables are as follows.

$X_{j}=\left\{\begin{array}{lr}1, & \text { if a facility is established at location } j \in J \\ 0, & \text { otherwise }\end{array}\right.$

$Y_{i j F_{s}}=\left\{\begin{array}{lc}1, & \text { if facility } j \text { serves c ustomer i in scenario } F_{s} \\ 0, & \text { otherwise }\end{array}\right.$

The RPMP model is

$$
\begin{gathered}
\operatorname{Min}_{s \in S}\left(\sum_{F_{s} \subseteq E_{s}} p_{F_{s}}\left(\sum_{i \in I} \sum_{j \in s} h_{i} d_{i j F_{s}} Y_{i j F_{s}}\right)\right) \\
\text { s.t. } \sum_{j \in s} Y_{i j F_{s}}=1, i \in I, s \in S, F_{s} \subseteq E_{s} \\
\sum_{j \in s} X_{j}=p \\
Y_{i j F_{s}} \leq X_{j}, i \in I, j \in J, F_{s} \subseteq E_{s} \\
X_{j} \in\{0,1\}, j \in J \\
Y_{i j F_{s}} \in\{0,1\} i \in I, j \in J, F_{s} \subseteq E_{s}
\end{gathered}
$$

Where $d_{i j F_{s}}$ is the distance of a shortest path between $i$ and $j$ in scenario $F_{s}$. The objective function minimizes the total expected operation cost of the system. Constraints (8) require that each customer to be served by exactly one facility in any edge failure case. Constraint (9) requires $p$ facilities to be opened. Constraints (10) require that any customer can't be assigned to an unopened facility in any edge failure case. Constraints (11) and (12) require the location variables and the assignment variables to be binary.

\section{An Instance and the Computing Results}

In this section we apply a data set to the model established in the last section to generate a reliable facility system. Our data set is derived from the 2008 China census data: a set of 34 vertices consisting of the capitals of all the provinces in China plus the two special administrative regions, Hong Kong 
and Macau. The demand of city $i$ is established to be the city's administrative region population divided by $10^{4}$. The links in the edge set are from the recent national highways and the transportation cost between city $i$ and city equals to the distance between the two cities.

We solve a 6-median problem in order to compare the solutions of the PMP and the RPMP model. The 6 suppliers are city 7 , city 10 , city 16 , city 17 , city 19 and city 23; the optimal value of the objective function is 573605 . The allocation plan is $7 \rightarrow 6,7,8 ; 10 \rightarrow 9,10,11,12 ; 17 \rightarrow 13,14,17,18,32$; $19 \rightarrow 19,20,21,33,34 ; 23 \rightarrow 22,23,24,25,26 ; 16 \rightarrow 1,2,3,4,5,15,16,27,28,29,30,31$.

This solution establishes a facility system shown in the Fig. 1, where the edges marked by red colour represent the delivery routes from each distribution centre to its customers.

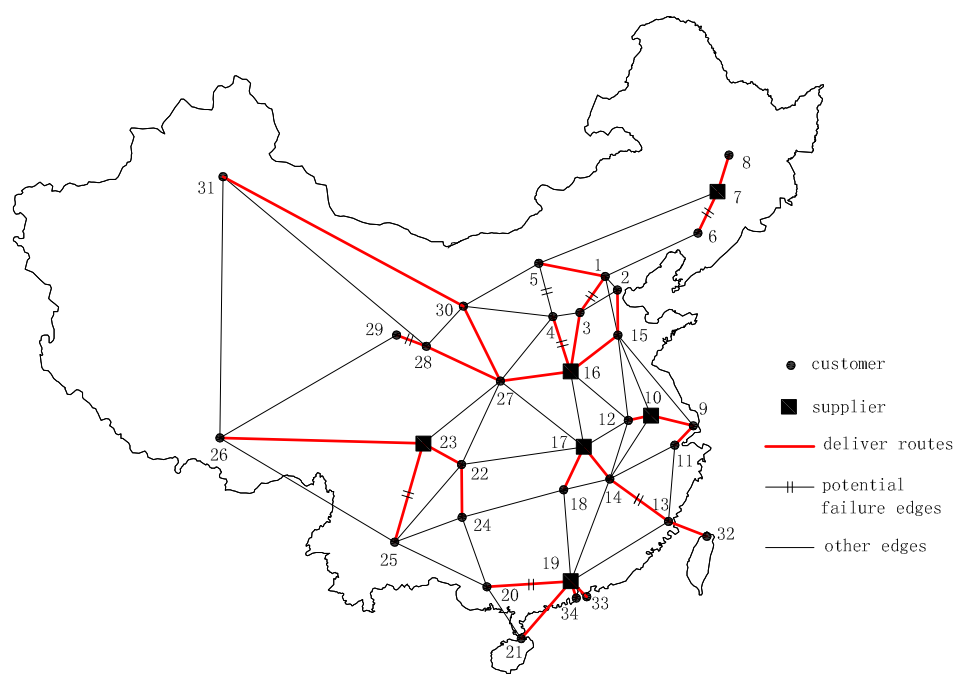

Fig. 1 The optimal solution of a 6-median problem

Given the failure edge set as the following table, without loss of generality, we estimate the edge failure probabilities according to the geographical environment and the history of the natural disasters. The data are shown as in the Table 1.

Table1. The Potential Failure Edges and the Probabilities

\begin{tabular}{|c|c|c|c|c|c|}
\hline Edge & Probability & Possible causes & Edge & Probability & Possible causes \\
\hline $1(4,5)$ & 0.2 & sandstorm & $5(23,25)$ & 0.3 & earthquake or flood \\
\hline $2(28,29)$ & 0.4 & snowstorm & $6(1,3)$ & 0.2 & congestion \\
\hline $3(6,7)$ & 0.5 & inclement weather & $7(19,20)$ & 0.3 & flood or typhoon \\
\hline $4(13,14)$ & 0.4 & inclement weather & $8(4,16)$ & 0.5 & congestion \\
\hline
\end{tabular}

Based on the optimal solution of the 6-median problem, we now select 10 points from $J$ as the potential suppliers of the RPMP: $J^{\prime}=\{1,3,7,10,14,16,17,18,19,23\}$. To do so can reduce the computing time without any impact to the optimality of the solutions.

We apply the above data to the RPMP model and obtain the optimal solutions as follows.

The 6 suppliers are $3,7,10,16,19,23$, and the objective function value is 615316 . It is easy to compute the total cost is 680293 in the worst case (all the edges in $F$ have failed).

However, if we don't consider the edge failures, the total cost of the optimal solution of the 6-median problem will be 786753 when the worst case occurs. 
As we can see, the optimal solutions of the reliable 6-median problem and the 6-median problem are very different. Firstly, the supplier 17 is replaced by 3 . Secondly, the supply-demand allocations are changed greatly. Finally, the expected objective value of the solutions of the 6-median problem under edge failure case is 619085 , greater than that of the former.

The deference between the objective function value of the optimal solution of the 6-median problem and the reliable 6-median problem is $619085-615316=3769$. The relative reliability (cost reliability) of the original solution (see Fig. 1) is $99.4 \%$. We also find that the solution of the reliable 6-median problem is more stable than that of the 6-median problem. This instance indicates that, considering the edge failure before making the location decisions, the system's reliability and stability can be increased obviously, although the total cost increase $7.3 \%$.

\section{Summary and Conclusions}

The concept of system reliability has not been defined properly since there are a variety of system functions. In this paper, we firstly propose the concept of cost reliability and consider the (cost) reliability of systems based on the solutions of the PMP when subject to edge failures.

The reliable p-median problem model established in this paper is realistic and useful. Although the instance we constructed is not large, the selection of the potential loss edge and the estimation of the edge failure probability are somewhat simple, it does not lose the generality and so the theoretical and practical significance are obviously.

Next, we will consider the reliability of systems based on other classical discrete facility location problems such as the uncapacitated fixed-charge location problem, the p-centre problem, and the set covering problem etc. with edge failures.

\section{Acknowledgment}

This paper was supported by the SXESF (No. 12JK0888 and 12JK0861). The authors are grateful to the anonymous referees of this paper.

\section{References}

[1] R. L. Church and M. P. Scaparra, Critical infrastructure, Springer Berlin Heidelberg, 2007, Chap. 11.

[2] Y. Sheffi, The resilient enterprise: overcoming vulnerability for competitive advantage, MIT Press, Cambridge, MA, 2005.

[3] M. Bundschuh, D. Klabjan and D.L. Thurston, Modeling robust and reliable supply chains, Work paper, University of Illinois, Urbana-Champaign, IL. 2003.

[4] L. V. Snyder and Z. J. M. Shen, Managing disruptions to supply chains, Forthcoming in The Bridge (National Academy of Engineering), 36 (2006) 39-45.

[5] S. L. Hakimi, Optimum location of switching centers and the absolute centers and medians of a graph, Operations Research, 12 (1964) 450-459.

[6] M. B. Teitz and P. Bart, Heuristic methods for estimating the generalized vertex median of a weighted graph, Operations Research, 16 (1968) 955-961.

[7] C. S. Revelle and R. Swain, Central facilities location, Geographical Analysis, 2 (1970) 30-42.

[8] R. L. Church, COBRA: A new formulation for the classic p-median location problem, Annals of Operations Research, 122 (2003) 103-120.

[9] L. V. Snyder and M. S. Daskin, Reliability models for facility location: the expected failure cost case, Transportation Science, 39 (3) (2005) 400-416.

[10]L. V. Snyder, M. P. Scaparra, M. S. Daskin and R. L. Church, Planning for disruptions in supply chain networks: In "Tutorials in Operations Research", Johnson, M. P., Norman, B. and Secomandi (eds.), N. Chap. 9, INFORMS, 2006. 